\title{
Is a more extensive operation justified for acute type $\mathrm{A}$ dissection repair?
}

\author{
Leonard N. Girardi, MD
}

From the Department of Cardiothoracic Surgery, Weill Cornell Medical College, New York, NY.
Disclosures: Author has nothing to disclose with regard to commercial support.
Received for publication Sept 15, 2018; accepted for publication Sept 17, 2018; available ahead of print Nov 3,
2018 .
Address for reprints: Leonard N. Girardi, MD, Department of Cardiothoracic Surgery, Weill Cornell Medical Col-
lege, 525 E 68 th St, M-424, New York, NY 10021 (E-mail: lngirard @ med.cornell.edu).
J Thorac Cardiovasc Surg 2019;157:12-3
0022-5223/ 36.00
Copyright @ 2018 by The American Association for Thoracic Surgery
https://doi.org/10.1016/j.jtcvs.2018.09.048

Matalanis ${ }^{1}$ summarizes the surgical techniques used and early results obtained in a very small cohort of patients undergoing total aortic repair for acute type A aortic dissection (ATAAD). Total aortic repair in his experience constitutes arch debranching and total arch replacement with creation of a long, polyethylene terephthalate segment that serves as a landing zone for subsequent distal endovascular reconstruction. Later during the index hospitalization or shortly after discharge, a covered endograft is inserted into the proximal descending aorta and the remainder of the thoracoabdominal aorta is treated with uncovered stents down to the aortic bifurcation. As with the Stent-Assisted Balloon-Induced Intimal Disruption and Relamination in Aortic Dissection Repair (STABILISE) concept ${ }^{2}$ ballooninduced intimal disruption is the vital, final step in this version of total aortic repair with the intent of inducing immediate intimal reapposition and eliminating false lumen (FL) hypertension. Complete FL thrombosis is the goal. Fifteen patients underwent this ultracomplex aortic procedure. Three patients with chronic dissections were subjected to total aortic repair between 3 and 7.5 years after their proximal operation and should be excluded from this report outlining a "new paradigm in the management of acute type A dissection." 1 Do the results in these 12 patients with ATAAD accomplish the author's goals of "yielding better outcomes both in the early phase as well as longer term?"1

Before contextualizing these results, it is crucial to better define indications for this Herculean task. Despite the author's obviously outstanding open surgical and endovascular skills, only $17 \%$ of patients with ATAAD (12 out of 72) were treated with total aortic repair. Patients with evolving malperfusion (none was present at the time of presentation but 2 developed limb malperfusion after stage I), rapidly enlarging FL on computed tomographic angiography (no data given on the delta from baseline), and those with a collapsed true lumen (no data given on the diameters of true lumen, FL, or total aorta) were deemed to be

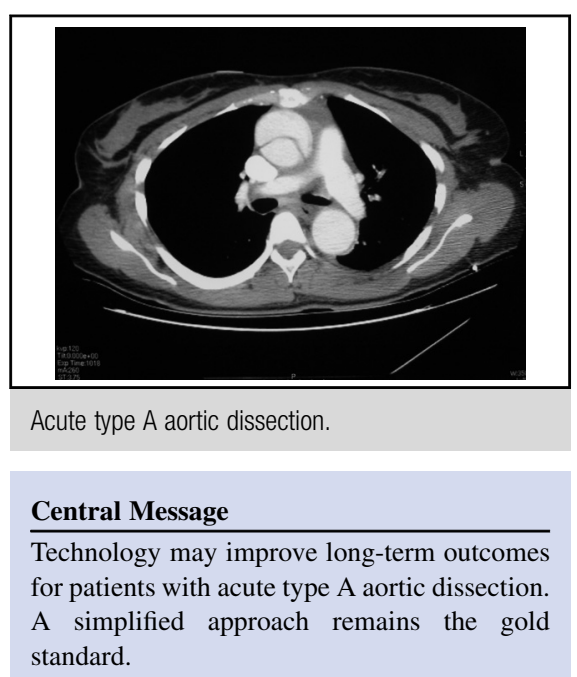

See Article page 3.

appropriate candidates. The immediate results were quite good with no operative mortality and an acceptable incidence of stroke (1 out of 15) and temporary hemodialysis (3 out of 15). Fortunately, unlike previous experiences with descending aortic coverage during ATAAD repair, in this very small cohort, there was no evidence of spinal cord injury. However, the need for early additional procedures was not insignificant. Forty percent required a visceral or renal artery stent. Twenty percent required an iliac stent. An additional $20 \%$ also required late reinterventions on the aorta within a median of 21 months during which follow-up computed tomography imaging was available. Only $53 \%$ (8 out of 15) had complete FL thrombosis. Late aortic dimensions were not provided for analysis.

The long-term influence on those $47 \%$ in this series with only partial FL thrombosis remains uncertain. Tsai and colleagues ${ }^{4}$ reported on 67 patients after "standard" acute type A repair ( 2 out of 3 underwent hemiarch and 1 out of 3 underwent total arch) who were followed for a mean of 6.3 years. Complete FL thrombosis was achieved in only $46.3 \%$ of patients, whereas partial thrombosis was evident in $32.8 \%$. A completely patent FL was seen in $20.9 \%$. Although those with a partially thrombosed FL had the fastest total aortic growth rate, only $8.9 \%$ (6 out of 67 ) of the entire cohort required a distal reoperation and the actuarial freedom from reoperation was $95 \%$ at 5 years and $87 \%$ at 10 years. There was no difference in the need for additional 
surgery between those with a completely thrombosed FL or a completely patent FL. Furthermore, 1-, 5- and 10-year survival was similar irrespective of the thrombosis status of the FL. We performed a meta-analysis of 7821 patients undergoing type A dissection repair and also found a reassuringly limited need for late reoperation after ATAAD. ${ }^{5}$ Only $11.5 \%$ of patients developed the need for either proximal or distal reoperation a mean of 5.2 years after their initial repair.

As with other interesting early experiments, these results generate many more questions than answers. The author appropriately cautions the need for larger numbers and longer follow-up before total aortic repair is more widely adopted. Only a well-designed, multi-institutional trial will be able to elucidate whether the benefits of highly complex early endovascular repair justify the additional risks and expenses when compared with simpler solutions for ATAAD.

\section{References}

1. Matalanis G, Ip S. A new paradigm in the management of acute type A aortic dissection: total aortic repair. J Thorac Cardiovasc Surg. 2019;157:3-11.

2. Hofferberth SC, Nixon IK, Boston RC, McLachlan CS, Mossop PJ. Stent-assisted balloon-induced intimal disruption and relamination in aortic dissection repair: the STABILISE concept. J Thorac Cardiovasc Surg. 2014;147:1240-5.

3. Leontyev S, Tsagakis K, Pacini D, Di Bartolomeo R, Mohr FW, Weiss G, et al. Impact of clinical factors and surgical techniques on early outcome of patients treated with frozen elephant trunk technique by using EVITA open stent-graft: results of a muticentre study. Eur J Cardiothorac Surg. 2016;49:660-6.

4. Tsai MT, Wu HY, Roan JN, Tsai YS, Hsieh PC, Yang YJ, et al. Effect of false lumen partial thrombosis on repaired acute type A aortic dissection. J Thorac Car diovasc Surg. 2014;148:2140-6.

5. Gaudino MF, Girardi LN, Rahouma M, Leonard JR, Di Franco A, Lau C, et al. Aortic re-operation after replacement of the proximal aorta: a systematic review and meta-analysis. Eur J Vasc Endovasc Surg. 2018;56:515-23. 